\title{
COVID-19: A Potential Cause of Non-convulsive Status Epilepticus
}

\author{
Karim El Aidaoui ${ }^{1}$, Rita Ait Benhamou ${ }^{1}$, Asmaa Hazim ${ }^{2}$, Amal Haoudar ${ }^{1}$, Chafik El Kettani ${ }^{1}$ \\ 1. Anesthesia and Critical Care, Cheikh Khalifa International University Hospital, Mohammed VI University of Health \\ Sciences, Casablanca, MAR 2. Neurology, Cheikh Khalifa International University Hospital, Mohammed VI University \\ of Health Sciences, Casablanca, MAR
}

Corresponding author: Karim El Aidaoui, kelaidaoui@um6ss.ma

\begin{abstract}
Coronavirus disease 2019 (COVID-19) has been described as being primarily responsible for respiratory symptoms. Although several case reports have shown the importance of neurological manifestations, only a few have reported non-convulsive status epilepticus (NCSE) as the first manifestation of COVID-19 infection. Here, we report the case of a 30-year-old male patient with no past medical history who was admitted with altered consciousness. On examination, the patient had a Glasgow Coma Scale (GCS) of 13/15. Vital signs were within normal range. Computed tomography scan of the and magnetic resonance imaging of the brain were normal. Biochemical assessments showed a mild hyponatremia $(134 \mathrm{mEq} / \mathrm{L})$ and high levels of D-dimer and lactate dehydrogenase. Urine drug screening did not find any abnormality and a lumbar puncture showed an increased cerebrospinal fluid protein. The result of the reverse transcription polymerase chain reaction test in the nasopharyngeal swab was positive for severe acute respiratory syndrome coronavirus 2 (SARS-CoV-2). Electroencephalogram (EEG) showed a generalized epileptiform activity. Upon undergoing antiepileptic treatment, patient's GCS improved to 15 gradually. A repeated EEG confirmed complete resolution of epileptic abnormalities four days later. This case report shows that SARS-CoV-2 infection can directly involve the central nervous system and can be manifested with isolated NCSE without any other neurological manifestations.
\end{abstract}

Review began 04/29/2021 Review ended 05/14/2021 Published 05/15/2021

(c) Copyright 2021

El Aidaoui et al. This is an open access article distributed under the terms of the Creative Commons Attribution License CC-BY 4.0., which permits unrestricted use, distribution, and reproduction in any medium, provided the original author and source are credited.
Categories: Neurology

Keywords: covid-19, status epilepticus, seizure, confusion, sars-cov-2

\section{Introduction}

Neurological manifestations associated with coronavirus disease 2019 (COVID-19) are frequent and varied. While non-specific symptoms such as headache, dizziness, and myalgia have been described, more severe neurological disorders have been reported in other patients [1]. We report the case of a non-convulsive status epilepticus (NCSE) in a young patient as the only neurological manifestation of COVID-19 infection.

\section{Case Presentation}

A 30-year-old male patient with no past medical history was admitted on November 30th, 2020 with altered consciousness. Three days prior to his admission, he felt unwell and feverish. There was no documented seizure, cough, or dyspnea. On examination, the patient was drowsy and confused. His Glasgow Coma Scale (GCS) was 13/15 (E3V4M6). Pupils were equal and reactive to light. There was no focal neurological deficit. No cardiorespiratory distress or head injury were noticed. The patient's vital signs were all within the normal range, including arterial pressure $(132 / 66 \mathrm{mmHg})$, heart rate (82 beats per minute), and temperature $\left(37.5^{\circ} \mathrm{C}\right)$.

The patient's sodium level was low (134 mEq/L), which could not explain his neurological symptoms. The blood sugar level was within normal limits $(156 \mathrm{mg} / \mathrm{dL})$. Hematological and inflammatory assessment showed a high level of D-dimer $(1,450 \mu \mathrm{g} / \mathrm{L})$ and lactate dehydrogenase $(317 \mathrm{U} / \mathrm{L})$ with normal C-reactive protein $(0.8 \mathrm{mg} / \mathrm{L})$, procalcitonin $(0.047 \mathrm{ng} / \mathrm{mL})$, ferritin $(166 \mu \mathrm{g} / \mathrm{L})$, and troponin $(0 \mathrm{ng} / \mathrm{mL})$. Urine drug screening did not find any abnormality. Computed tomography (CT) scan of the head showed a non-specific left cerebellum hypodensity. Magnetic resonance imaging (MRI) of the brain which was done to complete the investigation did not reveal any abnormality.

In the current pandemic context, a fever episode made us think of an infection with severe acute respiratory syndrome coronavirus 2 (SARS-CoV-2). Therefore, we performed a reverse transcription polymerase chain reaction (RT-PCR) test in nasopharyngeal swab and a chest CT. The chest CT did not show lesions compatible with COVID-19 infection. Subsequently, the patient was admitted in the neurological intensive care unit. A lumbar puncture was performed. Cerebrospinal Fluid (CSF) analysis showed the following results: CSF glucose at $86 \mathrm{mg} / \mathrm{dL}$, increased CSF protein at $54 \mathrm{mg} / \mathrm{dL}$, CSF white blood cell count at 8/ $\mathrm{mm}^{3}$, CSF red blood cells count at $5 / \mathrm{mm}^{3}$, with no organisms in the culture. Serology for human immunodeficiency virus, viral hepatitis, and syphilis were all negative. India Ink preparation for Cryptococcus was negative in CSF. Twenty- 
During the patient's hospital stay, hemodynamical and respiratory functions remained steady. However, his prolonged confusion remained unchanged. Therefore, a routine electroencephalogram (EEG) was performed, which showed a generalized epileptiform activity (Figure 1).

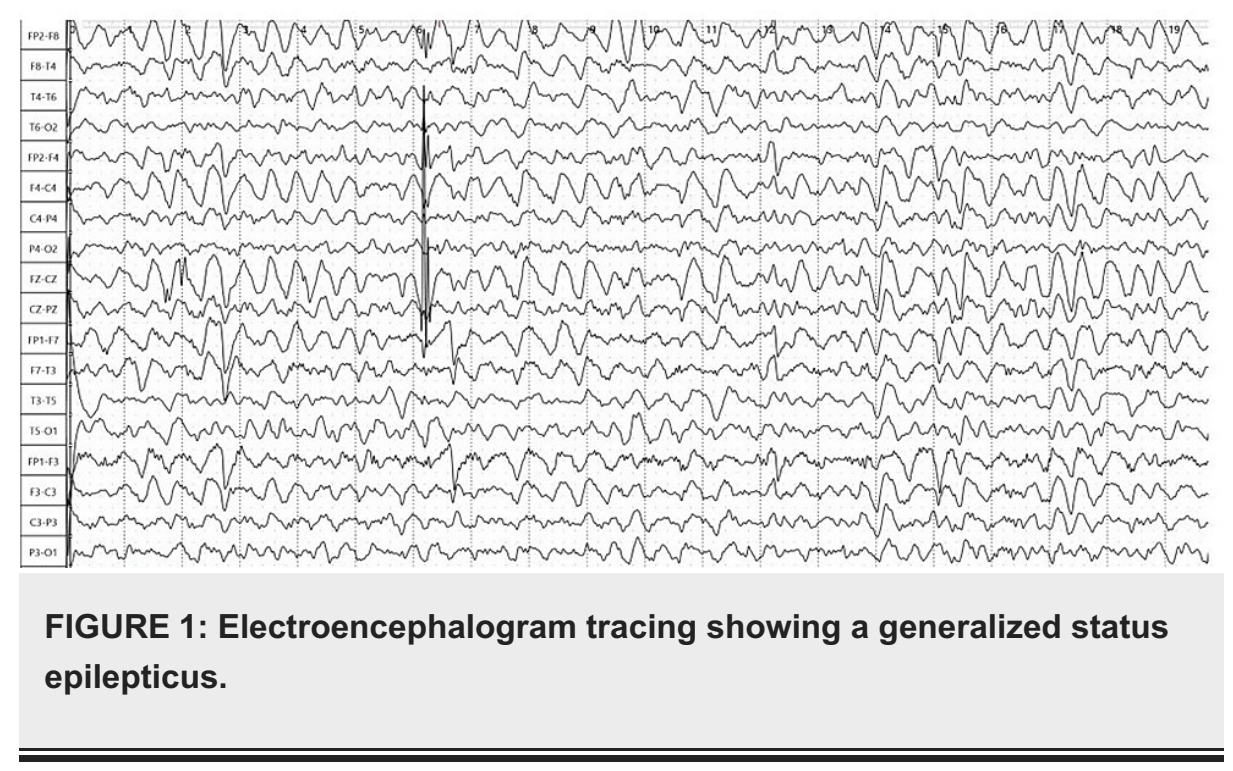

Upon undergoing antiepileptic treatment (midazolam $5 \mathrm{mg}$ intravenous followed by levetiracetam $0.5 \mathrm{~g}$ twice daily), the patient gradually improved. He was no longer confused 24 hours later. A follow-up EEG confirmed complete resolution of epileptic abnormalities after four days. The patient was discharged home after one week, without any recurrence of neurological symptoms during the follow-up of three months.

\section{Discussion}

In the beginning of the pandemic, COVID-19 was described as being primarily responsible for respiratory symptoms [2]. However, several studies have shown the existence of digestive, cardiac, and neurological manifestations [1,3]. Recently, neurological manifestations have been reported as an integral part of SARSCoV-2 infection symptomatology [4]. Mao et al. [2] investigated 214 COVID-19 patients, one-third of whom had neurological manifestations at admission, including confusion, ataxia, seizures, and acute cerebrovascular disease. The detection of the virus in CSF using PCR technique has been described in several publications [5]. Other reports confirmed COVID-19 infection based on IgM and IgG antibodies in CSF [6]. It has been shown that SARS-CoV-2 binds to angiotensin-converting enzyme 2 (ACE2) membrane receptors present in the pulmonary and vascular endothelium as well as neuronal cells. One of the preferred entry ways for virus in the brain can be a retrograde neuronal dissemination via the olfactory mucosa, followed by the olfactory bulb [7]. Other authors have suggested that the destructive effects of COVID-19 in the central nervous system would be caused by the entry of pro-inflammatory cytokines from the periphery into the central nervous system, or the production of cytokines by astrocytes and activated microglia. Proinflammatory cytokines can cause blood-brain barrier disruption, increase glutamate levels, reduce gammaaminobutyric acid (GABA) levels, and lead to epilepsy [8]. However, the exact way by which SARS-CoV-2 involves the central nervous and causes EEG abnormalities or seizures remains unclear $[7,8]$.

In fact, an isolated alteration of vigilance with a progressive change in consciousness ranging from somnolence to coma evokes an encephalopathy. Even if multifactorial origin encephalopathy related to hypoxia or multiorgan damage has been described in COVID-19 among severely ill patients admitted to the intensive care unit [9], our patient did not develop respiratory symptoms of COVID-19. This goes against this theory. In addition, our patient had no previous history of seizures, and the mild hyponatremia cannot explain seizure occurrence. The clinical presentation of our patient suggests a NCSE presenting as confusional state. NCSE represents a prolonged state of seizures without obvious motor activity [10]. In our report, it is a case of absence status (AS), which is the most common form of NCSE. AS is based on clinical, electrophysiological (EEG), and therapeutic criteria [11]. In our view, the patient had a primary central nervous system disease related to COVID-19 that led to NCSE. More studies are needed to demonstrate the exact mechanism of seizures in COVID-19 patients.

\section{Conclusions}

This case study reports a NCSE as the first manifestation of COVID-19 infection and shows that SARS-COV2 can enter the central nervous system directly and cause NCSE in susceptible patients. Hence, COVID-19 infection can be manifested with isolated NCSE without any other neurological manifestations. 


\section{Additional Information}

\section{Disclosures}

Human subjects: Consent was obtained or waived by all participants in this study. Conflicts of interest: In compliance with the ICMJE uniform disclosure form, all authors declare the following: Payment/services info: All authors have declared that no financial support was received from any organization for the submitted work. Financial relationships: All authors have declared that they have no financial relationships at present or within the previous three years with any organizations that might have an interest in the submitted work. Other relationships: All authors have declared that there are no other relationships or activities that could appear to have influenced the submitted work.

\section{References}

1. Mao L, Jin H, Wang M, et al.: Neurologic manifestations of hospitalized patients with coronavirus disease 2019 in Wuhan, China. JAMA Neurol. 2020, 77:683-90. 10.1001/jamaneurol.2020.1127

2. Zhu N, Zhang D, Wang W, et al.: A novel coronavirus from patients with pneumonia in China, 2019 . N Engl J Med. 2020, 382:727-33. 10.1056/NEJMoa2001017

3. El Aidaoui K, Haoudar A, Khalis M, et al.: Predictors of severity in Covid-19 patients in Casablanca, Morocco. Cureus. 2020, 12:e10716. 10.7759/cureus.10716

4. Ellul MA, Benjamin L, Singh B, et al.: Neurological associations of COVID-19. Lancet Neurol. 2020, 19:76783. 10.1016/S1474-4422(20)30221-0

5. Domingues RB, Mendes-Correa MC, de Moura Leite FBV, et al.: First case of SARS-COV-2 sequencing in cerebrospinal fluid of a patient with suspected demyelinating disease. J Neurol. 2020, 267:3154-6. 10.1007/s00415-020-09996-W

6. Wang M, Li T, Qiao F, Wang L, Li C, Gong Y: Coronavirus disease 2019 associated with aggressive neurological and mental abnormalities confirmed based on cerebrospinal fluid antibodies: a case report. Medicine (Baltimore). 2020, 99:e21428. 10.1097/MD.0000000000021428

7. Baig AM, Khaleeq A, Ali U, Syeda H: Evidence of the COVID-19 virus targeting the CNS: tissue distribution, host-virus interaction, and proposed neurotropic mechanisms. ACS Chem Neurosci. 2020, 11:995-8. 10.1021/acschemneuro.0c00122

8. Nikbakht F, Mohammadkhanizadeh A, Mohammadi E: How does the COVID-19 cause seizure and epilepsy in patients? The potential mechanisms. Mult Scler Relat Disord. 2020, 46:102535. 10.1016/j.msard.2020.102535

9. Helms J, Kremer S, Merdji H, et al.: Neurologic features in severe SARS-CoV-2 infection . N Engl J Med. 2020, 382:2268-70. 10.1056/NEJMc2008597

10. Kaplan PW: EEG criteria for nonconvulsive status epilepticus. Epilepsia. 2007, 48 Suppl 8:39-41. 10.1111/j.1528-1167.2007.01345.x

11. Genton P, Ferlazzo E, Thomas P: Absence status epilepsy: delineation of a distinct idiopathic generalized epilepsy syndrome. Epilepsia. 2008, 49:642-9. 10.1111/j.1528-1167.2007.01467.x 